Rapport - Société canadienne d'histoire de l'Église catholique

\title{
L'Épiscopat canadien et les bibliothèques paroissiales de 1840 à
}

\section{0}

\section{Antonio Drolet}

Volume 29, 1962

URI : https://id.erudit.org/iderudit/1007362ar

DOI : https://doi.org/10.7202/1007362ar

Aller au sommaire du numéro

Éditeur(s)

La Société canadienne d'histoire de l'Église catholique

ISSN

0318-6148 (imprimé)

1927-7075 (numérique)

Découvrir la revue

Citer cet article

Drolet, A. (1962). L’Épiscopat canadien et les bibliothèques paroissiales de 1840 à 1900. Rapport - Société canadienne d'histoire de l'Église catholique, 29, 21-35. https://doi.org/10.7202/1007362ar

Tous droits réservés @ La Société canadienne d'histoire de l'Église catholique, 1963
Ce document est protégé par la loi sur le droit d'auteur. L’utilisation des services d'Érudit (y compris la reproduction) est assujettie à sa politique d'utilisation que vous pouvez consulter en ligne.

https://apropos.erudit.org/fr/usagers/politique-dutilisation/ 


\section{L'Épiscopat canadien et les bibliothèques paroissiales de 1840 ò 1900}

Les bibliothèques paroissiales, au Canada, ont eu des débuts qu'il est difficile de retracer avec précision. Au temps du régime français, quelques curés, possesseurs de bibliothèques personnelles plus ou moins considérables, ont pu les mettre au service de leurs fidèles ${ }^{1}$. Un historien, l'abbé Auguste Gosselin, en parlant de l'époque de 1800 à 1820, à écrit qu'il se fondait un peu partout des bibliothèques dans les paroisses $^{2}$. Vers les années 1840, il est sûr qu'il en existait, du moins à quelques endroits.

Cependant, c'est à partir de 1845 que nous voyons les autorités religieuses annoncer leur intention d'établir un système de bibliothèques paroissiales. L'objet de notre étude est de faire connaître les intentions, les décisions épiscopales à ce sujet, ainsi que certaines circonstances où elles se placent et qui peuvent les expliquer.

Suivant une opinion partagée par plus d'un historien, avec les débuts de l'Union (1840), les Canadiens français ont commencé de connaître un renouveau, une renaissance dans à peu près tous les domaines : culturel, éducatif, nationaliste, politique, religieux ${ }^{3}$. Dans ce dernier domaine, particulièrement, ce renouveau, qui sembla aller de pair avec la conquête de la liberté politique, fut une véritable réaction contre une situation qui n'était pas sans inquiéter les autorités ecclésiastiques.

Certaines erreurs, aussi bien politiques que religieuses, avaient pénétré dans les esprits et amoindri la vie religieuse. Dans le remous des événements de 1837-1838, le peuple et une partie du clergé rural ${ }^{4}$, excités par un idéal trompeur de liberté, avaient laissé ébranler leur confiance dans leurs chefs religieux ${ }^{5}$. Un historien, parlant des années qui vont

1 J.-E. Roy, Histoire de la Seigneurie de Lauzon, 1897-1901, t. II:99-100.

2 L'abbé Auguste Gosseun, Le docteur Jacques Labrie, 1903, p. 91.

3 Cf. L'abbé Lionel Groulx, Histoire du Canada français, v. 3:307-312, ss.; abbé Marcel Dandurand, Les premières difficultés entre $M^{g r}$ Bourget et l'Institut Canadien de Montréal, Revue de l'Université d'Ottawa, 25:146, ss., 1955. Alphonse DésIlets, Les cent ans de l'Institut Canadien de Québec, Québec, 1949 , p. 67 , ss.

4 GrouLx, ouvr. cité, 3:217.

5 Fernand Oullet, Le mandement de $M^{g r}$ Lartigue et la réaction libérale, Bulletin des recherches historiques, 52:104, 1952; Léon Pouliot, s.j., $M^{\text {or }}$ Bourget et son temps, Montréal, Beauchemin, 1955, t. I:137, 141, passim; GroulX, ouvr. cité, 3:190, 216, 217, 218. 
de 1837 à 1840, les appelle « les années terribles du diocèse de Montréal ${ }^{6}$.

Ce fut sans doute le grand mérite de $\mathrm{M}^{\text {gr }}$ Ignace Bourget, à son entrée en fonction comme deuxième évêque de Montréal (en 1840), d'avoir compris combien la situation religieuse était critique, et d'avoir posé les bases d'une véritable renaissance religieuse, en tirant plein parti d'un climat nouveau où l'Eglise canadienne réapprit à se servir d'une liberté presque oubliée depuis la conquête ${ }^{7}$. Il renforcit les cadres de l'organisaiiun eciclésiastiqưue eñ recrutant jusqu'en Europe des effectifs destinés à accroître un clergé par trop insuffisant. Il fit venir des congrégations d'hommes au pays, les Jésuites y revinrent prendre une place que les Canadiens leur avaient conservée dans leur souvenir, des congrégations féminines nouvelles furent fondées ${ }^{8}$. Des grandes missions pastorales atteignirent toutes les classes de la population, clergé et fidèles, dans toutes les régions des villes et des campagnes ${ }^{9}$. Sous l'égide de $\mathbf{M}^{\mathrm{gr}}$ Bourget, un premier journal catholique vit le jour, les Mélanges religieux. Dirigé et rédigé de l'évêché, il servit d'organe de défense religieuse, dans une complète indépendance des partis politiques ${ }^{10}$. C'est dans un tel contexte de réforme religieuse que se situent les initiatives par lesquelles l'autorité ecclésiastique entreprit d'établir un réseau de bibliothèques sous sa direction.

Mais avant de faire connaître l'importance et le nombre des mesures prises à ce propos, il n'est pas sans intérêt d'essayer de retracer quelles furent les raisons qui ont pu motiver ce qu'on peut appeler le mouvement des bibliothèques paroissiales. Ce n'est certes pas par hasard que, pendant plusieurs années, l'épiscopat s'intéressa aux bibliothèques paroissiales; c'est sans doute que leur nécessité s'était révélée comme reliée à des besoins réels et bien précis, ressentis par le peuple autant que par ses chefs religieux ${ }^{11}$.

On sait qu'une des conséquences de la conquête avait été l'entrée au pays d'un élément protestant. Rattaché de près au nouveau pouvoir politique, imbu de la valeur de ses croyances, cet élément se trouva dans une situation favorable pour essayer de les implanter chez les Canadiens ${ }^{12}$. L'école, qu'on pensa d'abord utiliser comme un moyen efficace de conversion, ne donna pas les résultats espérés ${ }^{13}$ et le prosélytisme protestant dut se tourner vers d'autres moyens et, particulièrement, la propagande par l'imprimé ${ }^{14}$.

6 Léon Pouliot, s.j., Les dernières années et la survie de Mor Bourget, 1876 1885, Montréal, Beauchemin, 1960, p. 11.

7 Poulot, ouvr. cité, 1:155, ss., 2:26, 49, 51. Groulx, ouvr. cit., 3:317, 318. Poulrot, ouvr. cité, 2:64.

9 Groulx, 3:309, 317; Pouliot, 2:23, 37, 39, 142, 144.

10 Dandurand, ouvr. cité, 161.

11 M. Dandurand, ouvr. cité, pp. 146, 147.

12 L'abbé Arthur Mareux, Le problème protestant, étude lue au Congrès de la Société canadienne d'histoire de l'Eglise, octobre 1940, Québec, 1941, p. 3.

13 Frère Robert Sylvain, Aperçu sur le prosélytisme protestant au Canada français de 1760 à 1860, M.S.R.C., $3^{\circ}$ s., 55, I:67, 69, 1961.

14 Роulot, ouvrage cité, 2:11. 
Cette propagande se fit d'abord par la Société de la Bible (1804), établie au Canada peu après sa fondation en Angleterre ${ }^{15}$, et vouée spécialement à la diffusion du texte protestant des Livres saints et de tracts religieux. A partir de 1839 apparut une autre société, formée d'éléments dissidents de l'anglicanisme et fondée expressément pour travailler à la conversion des Canadiens français, la French Canadian Missionary Society, qui entreprit un travail systématique de propagande. Un troisième organisme, la Société missionnaire du nouvel évangile, se donna pour tâche « d'annoncer la bonne nouvelle du salut à ces pauvres Canadiens français, que leurs prêtres tenaient dans un cruel état d'ignorance ${ }^{16}$. Un peu plus tard, en 1851, parut un journal protestant de langue française, Le semeur canadien ${ }^{17}$. En 1856, les Anglicans entrent à leur tour en lice avec The Church of England Mission to the Frenchspeaking population of British North America ${ }^{18}$.

On peut ajouter que la propagande aurait pu tirer certain avantage du fait que des prêtres canadiens-français passaient de temps à autre au protestantisme ${ }^{19}$.

C'est donc particulièrement par l'imprimé - bibles, tracts, journal - que les groupements protestants entreprirent leur campagne de conversion. Un chiffre peut donner une certaine idée de l'importance des moyens mis en ouvre : en 1842, la Société de la Bible établissait à 54,478 le nombre de bibles qu'elle avait distribuées dans la région de Montréal ${ }^{20}$. Cette forme de prosélytisme, qui se continua à travers les années, devait nécessairement susciter une réaction catholique, et comment mieux répondre à l'imprimé que par l'imprimé, au livre, par le livre? Un autre motif devait s'ajouter à la nécessité de faire échec à la propagande protestante et libertaire, une raison positive, celle de procurer de la lecture conforme aux principes catholiques à une population qui, de plus en plus nombreuse, avait accédé à l'instruction primaire. Sans doute, existait-il déjà quelques bibliothèques ${ }^{21}$ destinées à un certain public, tôt apparues sous le régime anglais, mais l'élément de langue française qui pouvait les fréquenter s'y trouvait exposé à une influence nécessairement protestante, ou à tout le moins, areligieuse et rationaliste, puisque ces bibliothèques étaient dirigées par une majorité de langue anglaise, imbue des idées des philosophes du XVIII ${ }^{\bullet}$ siècle ${ }^{21}$.

Il apparaît clairement dans les divers documents épiscopaux que ces motifs furent à l'origine de l'action de la hiérarchie en vue de l'établissement de bibliothèques vraiment conformes aux besoins des catholiques.

15 La Société de la Bible fut établie à Montréal en 1823 et à Québec en 1835.

A Toronto, c'est en 1818 qu'elle apparut.

16 Mélanges religieux, 2:324, 12 novembre 1841.

17 Sylvarn, ouvrage cité, p. 72.

18 Id., p. 73.

19 Sylvain, ouvrage cité, p. 71.

20 Mélanges religieux, 4:15, 1842. $\mathrm{N}^{\circ} 12$, Montréal, 1947, p. 9.16.

21 E.-Z. MAssicotre, Bibliothèques d'autrefois à Montréal, Les Cahiers des Dix. 21. Idem. 
Sans doute, les bibliothèques ne furent pas le seul moyen employé pour faire échec à la propagande protestante par l'imprimé. On voulut, par exemple, faire face à la distribution de la Bible par la diffusion d'une traduction du Nouveau Testament, faite par $M^{\mathrm{gr}}$ Baillargeon, évêque de Québec. Monseigneur Bourget écrivit à ce propos : \& L'on me demande depuis longtemps de donner au peuple le Nouveau Testament en français afin d'empêcher de lire les bibles falsifiées. Je me rends aux instances qui m'ont été faites là-dessus. Nous allons donner le Nouveau Testament en langue vulgaire, lequel pourra être un livre d'ćcole ${ }^{22}$. : C'ćtait, de ecttc façon, retourner contre l'adversaire l'armo même dont il se servait. De même, la fondation de journaux catholiques donna des organes autorisés à prendre la défense des principes catholiques contre les idées pernicieuses de toutes sortes : apparaissent successivement les Mélanges religieux, en $1840^{23}$, le True Witness, en $1850^{24}$. Cependant, c'est à un rôle d'importance que $\mathrm{M}^{\mathrm{gr}}$ Bourget destinait les bibliothèques. Dans son Mandement d'installation de l'OEuvre des bons livres, il donnait pour but à cette œuvre : "défendre la foi et les mœurs attaquées par des productions impies et immorales ${ }^{25}$. L'ordonnance épiscopale de l'organisation de cette œuvre spécifie. à l'article V, que "le but de l'Association est de combattre l'impiété en opposant, aux livres impies, des livres pleins de la doctrine de la foi et conformes à ses dogmes et à sa morale » ${ }^{26}$.

En 1850, lorsque tout l'épiscopat prend officiellement position au sujet des bibliothèques paroissiales, donnant ainsi suite aux initiatives déjà prises par l'évêque de Montréal, la circulaire publiée par les évêques s'exprime comme suit :

"Il est évident que, pour éloigner le peuple des mauvaises lectures. il importe de lui procurer les moyens d'en faire de bonnes. car il faut un remède approprié pour chaque maladie ${ }^{27}$. 》

En mai 1854, les Pères du deuxième Concile provincial de la province de Québec écrivaient aux fidèles : "Il ne suffit pas de procurer l'instruction chrétienne à vos enfants pendant leur premier âge; vous aurez encore à leur fournir les moyens d'étendre les connaissances acquises à l'école... Il serait inutile, même dangereux, de répandre l'instruction primaire, si on négligeait les moyens de la rendre fructueuse. Or, rien n'est plus propre à conduire à ce but que la création de bonnes bibliothèques paroissiales."

(En passant, il n'est pas sans intérêt de rapprocher cette affirmation, de celle que fera plus tard un homme politique français, d'idée très différente, un temps ministre célèbre de l'Instruction publique. Jules

Circulaire, 16 février 1843; Mandements, dioc. de Montréal, I:236.

23 Pounot, ouvrage cité, II:34, 36, 37, 19.

24 Mandements, Montréal, II:353; Circulaire, 21 déc. 1850.

25 Mandements, Montréal, I:304, 1845.

26 Mandements, Montréal, I:306, sept. 1845.

27 Circulaire, 11 mai 1850; Mandements, Montréal, II:115. 
Ferry (1880), qui a écrit : "On pourra tout faire pour l'école et le lycée, si l'on n'organise pas de bibliothèques, on n'aura rien fait ${ }^{28}$. ")

En 1857, $\mathrm{M}^{\mathrm{gr}}$ Baillargeon, évêque de Québec, reviendra pour son compte sur le sujet : " On peut dire que les bibliothèques de paroisses sont un complément nécessaire de nos écoles. Pour que nos jeunes gens complètent l'instruction qu'ils ont reçue dans ces écoles, il faut qu'ils aient des livres... Si l'on ne veut pas qu'ils en lisent de mauvais, il faut leur en procurer de bons. Et ce n'est qu'en établissant des bibliothèques à leur usage dans chaque paroisse que l'on réussira à leur procurer des livres ${ }^{29}$."

L'urgence de procurer de bonnes lectures aux fidèles, et particulièrement aux jeunes, s'avérait d'autant plus pressante que, de toute évidence, la distribution des publications protestantes se révélait considérable et que le nombre de personnes que pouvait atteindre la propagande imprimée augmentait d'année en année, à mesure que diminuait l'analphabétisme. Quelques chiffres peuvent suffire pour faire apprécier les progrès obtenus après 1840 dans l'instruction populaire; ils sont d'autant plus significatifs que, peu d'années auparavant, soit en 1825, un écrivain canadien, Michel Bibaud, évaluait au chiffre peu élevé de 13,100 personnes le nombre de Canadiens français qui savaient lire ${ }^{30}$.

Quelques années plus tard, en 1836, le Bas-Canada pouvait compter 38,377 écoliers; ce nombre était porté à 61,030 en 1844, à 79,284 en $1851^{31}$, pour atteindre successivement 127,058 en 1855 , et 172,155 en $1860^{32}$. La progression dans la fréquentation des écoles s'accélère rapidement après 1840 et dépasse, proportionnellement, l'augmentation de la population. C'était sans doute un résultat encourageant pour ceux qui se préoccupaient de l'instruction populaire. Aux yeux des dirigeants ecclésiastiques devait se poser, de plus en plus aigu, le problème de fournir de bonnes lectures à une population de plus en plus nombreuse, exposée aux dangers de la propagande des mauvaises doctrines.

A lire les mises en garde, les conseils de prudence qui se répètent, de 1840 à 1900 , dans les mandements et les circulaires des évêques, soit qu'ils s'adressent individuellement ou collectivement à leurs fidèles, on ne peut manquer de conclure que cette propagande constituait une préoccupation qu'on pourrait qualifier de permanente.

C'est $\mathrm{M}^{\mathrm{gr}}$ Bourget qui ouvre la voie des recommandations, des défenses, peu après 1840, par une première lettre pastorale en date du 12 avril 1841. Les évêques de la province de Québec en font état dans leur lettre collective du 11 mai 1850. Les Pères des deuxième

A. Masson et P. Salvan, Les Bibliothèques, Paris, Presses Universitaires, Coll. Que Sais-je? p. 23.

29 Circulaire, 29 janv. 1857; Mandements, évêques de Québec, IV:293.

30 M. Bibaud, Bibliothèque canadienne, t. I, 1825, p. 119.

31 Groulx, Histoire du Canada français, 3:309.

32 Cf. Meilleur, Mémorial sur l'éducation du Bas-Canada, Montréal, 1860. 
$(1854)^{33}$, troisième $(1863)^{34}$, quatrième $(1868)^{35}$ et septième conciles ${ }^{36}$ provinciaux consacrent, à la question de la propagande protestante, plusieurs pages de leurs communications aux fidèles.

On en trouve des échos, en 1868, chez l'évêque de Saint-Hyacinthe ${ }^{37}$, de même que sous la plume de $\mathbf{M}^{\text {gr }}$ Taschereau, archevêque de Québec, et de $\mathrm{M}^{\mathrm{gr}}$ Bégin, son successeur ${ }^{38}$. Il arrive que certains conseils que donnent les pasteurs sur la façon de se débarrasser des publications dangereuses, sont suivis à la lettre; des autodafés provoquent des réactions dans les milieux protestants auxquels font écho les journaux ${ }^{39}$. A la suite de dénonciations faites par les curés, on saisit livres et gravures obscènes ${ }^{40}$.

La surveillance de la mauvaise littérature ne néglige pas le commerce de librairie. En 1857, le libraire canadien-français Rolland, de Montréal, fait détruire 1,500 volumes, après les avoir fait censurer par un prêtre du Séminaire ${ }^{41}$. Ce dernier fait est une preuve que ce n'est pas seulement du côté protestant que le danger pouvait provenir d'une certaine façon le danger protestant était bien connu, ce qui le rendait peut-être moins menaçant : "Nous ne parlons pas de certaines publications protestantes... ces feuilles portent, dans leurs titres mêmes et dans la franchise de leurs attaques, le contrepoison de leur pernicieuse doctrine ", comme s'expriment les Pères du quatrième concile provincial de Québec ${ }^{42}$.

C'est sans doute un ennemi plus pernicieux, plus subtil, qui attire l'attention des hommes d'Eglise, celui qui origine de l'intérieur, du milieu de langue française et, du moins par le baptême, de foi catholique. Certains éléments antireligieux, anticléricaux, se révèlent, dans des groupements comme l'Institut Canadien, de Montréal ${ }^{43}$; leurs idées se font jour dans des publications comme les journaux L'Avenir et Le Pays, où l'on rejette certaines prescriptions de la morale catholique et de l'Eglise. A maintes reprises, les évêques, et tout particulièrement $\mathrm{M}^{\mathrm{gr}}$ Bourget, lancent des mises en garde contre les mauvais journaux.

L'Institut Canadien et ses publications seront mis à l'index ${ }^{44}$. Plus tard, des condamnations seront portées contre des journaux ${ }^{45}$, Le Pays ${ }^{46}$, le Daily Witness, en $1875^{46^{a}}$; Le Courrier des Etats-Unis, en

Mandements, diocèse de Québec, 4:167, ss.

Id., p. 447, ss.

Id., p. 617, ss.; Mandements, diocèse de Saint-Hyacinthe, 3:60, ss.

Lettre pastorale des Pères du VII' Concile provinciale de Québec, 6 juin 1886;

Mandements des évêques de Chicoutimi, Chicoutimi, 1883, v. I:439.

Mandements, diocèse de Saint-Hyacinthe, Montréal, 1888, v. III:10.

Mandements, dioc. de Québec, 7:73, 8:93, etc.

Mélanges religieux, $5: 357,1842-1843$.

Le Canadien, Québec, 17 mai 1843.

Séraphin Marion, Les lettres canadiennes d'autrefois, Ottawa, Ed. de l'Université d'Ottawa, 1944, t. 4:28, 29; Auguste ViatTe, Histoire littéraire de l'Amérique française, Québec, 1954, p. 95.

Mandements, diocèse de Montréal, 5:329.

Dandurand, ouvrage cité, pp. 150, 151, 156, 157, 160.

Mandements, Montréal, VI:23.

Dandurand, ouvrage cité, p. 293.

Mandements, Montréal, IV-111.

Idem. 
$1882^{4 i}$, une réédition de la Lanterne, du publiciste Arthur Buies, en $1886^{48}$, par $\mathrm{M}^{\mathrm{gr}}$ Taschereau, évêque de Québec. En 1892, autres condamnations de Canada Revue et de l'Echo des Deux-Montagnes, par l'évêque de Montréal ${ }^{49}$. A l'occasion des mises en garde et des condamnations, on rappelle le rôle important des bibliothèques paroissiales ${ }^{50}$.

On peut observer qu'au problème religieux de la nécessité de bibliothèques conformes aux idées catholiques, pouvait s'ajouter, pour les fidèles de langue française, celui un peu différent, bien que connexe, de la nécessité de bibliothèques de langue française dirigées par eux. Au début de l'Union, nous l'avons noté déjà, les Canadiens français ne possédaient aucune bibliothèque de caractère public ${ }^{51}$.

Celles qui existaient jusqu'alors, les Instituts des Artisans, déjà très répandus, certaines sociétés qui offraient à leurs membres des facilités de lecture, avaient été fondés et étaient dirigés par des gens en majorité de langue anglaise et le choix des ouvrages qu'on y réunissait ignorait évidemment les prescriptions de la morale catholique dans ce domaine et étaient surtout des publications de langue anglaise.

Pour énumérer, en les résumant, les motifs qui peuvent expliquer et justifier les initiatives de l'autorité ecclésiastique, on peut donc citer la lutte contre la propagande protestante par les imprimés, la défense des idées catholiques en face des erreurs de toutes sortes, la nécessité de continuer l'œuvre d'instruction commencée par l'école, en somme, un ensemble de besoins propres à une population catholique surtout de langue française, jusqu'alors plutôt dépourvue de moyens de culture et vivant dans le voisinage d'une population anglaise déjà mieux outillée à ce sujet. C'est sans doute par une prise de conscience de cette situation que les évêques furent amenés à susciter des bibliothèques qui favoriseraient leur travail apostolique et apporteraient, en même temps, une réponse au besoin de lectures et de connaissances, besoin peut-être éveillé par une propagande inopportune, mais qu'il n'en était pas moins nécessaire de satisfaire pour faciliter l'instruction du peuple.

Dans le mouvement religieux de l'époque, l'action en faveur des bibliothèques sous une direction catholique pouvait aussi marquer, sous un aspect plus général, un pas de plus dans la libération de l'élément canadien-français, qui avait subi une certaine sujétion à l'élément de langue anglaise, le seul, jusqu'alors, à avoir pris des initiatives sérieuses dans le domaine des bibliothèques populaires.

Nous avons vu que la fondation des Mélanges religieux, entreprise de presse catholique, avait procuré à l'évêque de Montréal un organe où pouvait librement s'exprimer l'opinion catholique.

Il n'est donc pas étonnant que c'est d'abord dans ce journal que soit apparue une étude qui se révèle comme une première esquisse des

Mandements, Québec, VI:324.

48 Mandements, Québec, VI:591.

49 Mandements, Montréal, XI:107; Circulaire, 11 nov. 1892.

$50 \mathrm{M}^{\mathrm{gr}}$ Ignace BourGer, Circulaire, 19 mai 1852.

51 A. DésIlets, Les 100 ans de l'Institut Canadien de Québec, Québec, 1949, p. 69. 
projets, au sujet des bibliothèques paroissiales, que devaient élaborer par après les documents épiscopaux officiels.

Alors que le mandement de $\mathrm{M}^{\mathrm{gr}}$ Bourget, sur l'installation de $\mathrm{r} O E u$ vre des bons livres, est daté du 20 septembre 1845, c'est dès le 25 mars 1843 que les Mélanges religieux présentent un plaidoyer sur l'utilité des bibliothèques paroissiales ${ }^{52}$. Cet exposé parut sous une manchette intitulée Bulletin. Sous ce titre, la rédaction présentait couramment des commentaires et des études sur des sujets variés. Si l'on avait voulu donner un titre approprié au bulletin du 25 mars 1843 , on aurait pu. suivant la phraséologie de l'époque, lui donner comme en-tête : "Considérations sur la nécessité actuelle de l'instruction par la lecture et des bibliothèques comme moyen de répondre à cette nécessité.»

L'auteur ne s'y est pas limité à des observations générales sur la nécessité de l'instruction, il a recouru à des affirmations catégoriques, dont on pressent qu'elles avaient pour lui, et ses lecteurs, des incidences relatives au milieu canadien-français de son temps. En voici quelques extraits significatifs :

Les tems (sic) où l'ignorance était en quelque sorte permise... sont loin de nous... Il faut être de son siècle, marcher avec son siècle, si l'on n'en veut pas être l'enfant perdu et abandonné... Personne ne saurait sans danger demeurer oisif et stationnaire dans un tems (sic) d'activité et de progrès... les retardataires seraient infailliblement perdus... L'ignorance est un déshonneur et presqu'un crime aux yeux de la civilisation actuelle.

Et voici une observation qui touchait sans doute au vif des problèmes de l'élément canadien-français en contact avec une population différente, active et progressive :

Les progrès de l'éducation chez quelques peuples, chez certaines classes de la société ont imposé aux autres non plus la convenance, mais la nécessité de les suivre dans la voie de la connaissance et de l'instruction.

De ce plaidoyer pour l'instruction, conçu et écrit sous les yeux de l'évêque de Montréal, tout serait à citer, pour la signification qu'il pouvait prendre à l'époque, non moins que pour une actualité qu'il n'a pas perdue. Cependant, l'argumentation de l'auteur avait un but bien précis, et c'était de mettre en valeur, d'affirmer le rôle primordial de la lecture et des bibliothèques, comme moyen de faire accéder le peuple à l'instruction :

La lecture, une lecture sage et ordonnée, peut seule nous initier à ces connaissances plus que jamais une nécessité pour tous... Depuis longtemps on sent le besoin de bibliothèques paroissiales, où toutes les classes, tous les sexes et tous les âges peuvent aller chercher des distractions, de l'instruction, de l'édification.

L'auteur ne s'en tient pas à cette constatation, évidente à ses yeux; après avoir cité l'exemple de quelques bibliothèques déjà en existence. il indique de quelle façon pratique on peut mettre sur pied une bibliothèque paroissiale : réunion d'une assemblée des paroissiens, souscrip- 
tion et abonnements à recueillir, formation d'un comité sous la direction immédiate du curé. Tous ces détails seront repris, élaborés, précisés dans des documents officiels ultérieurs, mais déjà l'essentiel s'y trouve : raisons de fonder des bibliothèques, et moyens pour les établir et les maintenir.

La série des documents qui va reprendre le sujet de l'article des Mélanges religieux, c'est par un mandement de $\mathbf{M}^{\mathrm{gr}}$ Bourget qu'elle débute. Publié le 20 septembre 1845, il s'intitule Mandement d'installation de l'Euvre des bons livres, et il est en somme la charte de l'institution des bibliothèques comme une œuvre officiellement reconnue. L'évêque y rappelle d'abord que l'CEuvre des bons livres, inaugurée à Montréal l'année précédente, a déjà donné des résultats encourageants : une bibliothèque de 4,000 volumes a été formée et est fréquentée par 4.00 abonnés. Après avoir affirmé et énuméré les avantages d'une telle œuvre, l'évêque statue et ordonne les articles suivants :

1) l'approbation du diplôme de l'Archiconfrérie des bons livres, par lequel l'œuvre de Montréal est unie à celle de Bordeaux (France) déjà approuvée par le Pape;

2) l'approbation des règlements de l'Archiconfrérie;

3) la reconnaissance des indulgences attachées à l'œuvre;

4) le chant du Veni Creator, à la cathédrale, pour marquer l'installation de l'œuvre dans le diocèse de Montréal.

Le document épiscopal donne ensuite l'ordonnance d'organisation et les règlements de l'œuvre : elle devient un organisme ecclésiastique, sous la direction de l'évêque et des curés, aidés d'un comité, et les détails d'organisation, de formation et de fonctionnement sont déterminés par l"és êque lui-même.

Dans les années qui suivent, d'autres documents étendirent aux autres diocèses l'initiative prise par $\mathbf{M}^{\mathrm{gr}}$ Bourget pour le sien. Par leur circulaire du 11 mai 1850, les évêques des diocèses de Québec et d'Ontario recommandent l'établissement de bibliothèques dans chaque paroisse ${ }^{53}$ : ils annoncent qu'une commission a été chargée de préparer un catalogue des meilleurs livres propres à former une bibliothèque et de s'enquérir des moyens les moins coûteux pour en faire l'acquisition. La commission comprend des prêtres des diocèses de Montréal, d'Ottawa, de Kingston et de Québec. De ce dernier diocèse, c'est l'abbé CharlesFrançis Baillargeon, futur coadjuteur, à ce moment curé de la paroisse Notre-Dame de Québec, où il avait déjà, en 1843, fondé une bibliothèque paroissiale ${ }^{54}$.

Cette circulaire des évêques est une reconnaissance collective de l'Cuvre des bons livres, déjà reconnue par $\mathrm{M}^{\mathrm{gr}}$ Bourget en 1845. Une

53 Vandements, dioc. de Québec, 3:569, ss.; Mandements, lettres pastorales et circulaires des évêques de Saint-Hyacinthe, Montréal 188, v. I:490, ss.

it Mélanges religieux, 3:250, 1842; A. DrolET, Une bibliothèque française ̀̀ Québec en 1843, Cahiers de la Société historique de Québec, $n^{\circ} 13$, Québec, 1961. pp. $16-25$. 
lettre pastorale des évêques de la province ecclésiastique de Québec accompagne la circulaire que nous venons de citer, par laquelle l'Euvre des bons livres est établie dans chaque paroisse. Voici un passage de cette lettre qui éclaire sur la pensée et la conviction des évêques :

Par cette institution canonique, chaque bibliothèque sera une aut 1 . chaire de vérité dont les livres seront comme autant de langues de feu qui iront se poser sur chacune de vos maisons.

En 1852, une décision promulguée par l'évêque de Montréal montre que la bibliothèque paroissiale est une des genvres cur lesquelles chaque curé doit faire rapport chaque année à son évêque.

L'année suivante, en 1853, la bibliothèque paroissiale est un sujet d'étude imposé pour une "conférence ecclésiastique ", réunion périodique où les membres du clergé paroissial traitaient de sujets d'intérêt pastoral ${ }^{5 \overline{5}}$.

En 1854, la lettre pastorale des Pères du deuxième Concile provincial de Québec rappelle l'importance de compléter l'instruction des jeunes par la création de bibliothèques, comme, insiste le document, " on en trouve déjà dans plusieurs paroisses ". " Il serait inutile, même dangereux de répandre l'instruction primaire, si on négligeait les moyens de la rendre fructueuse ", déclarent les évêques ${ }^{56}$.

Au cours de 1855, une circulaire officielle de $\mathbf{M}^{\mathrm{gr}}$ Bourget publie de nouveau les règlements de l'CEuvre des bons livres, promulgués une première fois dix ans auparavant, en 1845 , avec le Mandement d'installation de l'œuvre. ${ }^{57}$.

A Québec, dans une circulaire du 21 juin 1857, $\mathbf{M}^{\mathrm{gr}}$ Baillargeon fait état d'un Indult par lequel le pape Pie IX accordait de nouvelles indulgences à l'Euvre des bons livres ${ }^{58}$; l'évêque rappelle la nécessité des bibliothèques paroissiales : "Elles sont, écrit-il, le complément nécessaire de nos écoles." Il réfère ses lecteurs à la circulaire collective des évêques (11 mai 1850) sur le sujet, et expose les raisons qui motivent les encouragements du pape en faveur de l'œuvre.

Dès les débuts de son épiscopat, en 1867, le premier évêque de Rimouski, $M^{\text {gr }}$ Jean Langevin, place, dans ses ordonnances épiscopales. la bibliothèque paroissiale parmi les œuvres dont les curés doivent s'occuper et sur laquelle ils doivent faire rapport annuellement ${ }^{59}$.

En 1871, il revient sur le sujet dans une circulaire et une lettre pastorale : "Les bibliothèques paroissiales, écrit-il, sont un moyen puissant d'entretenir chez les enfants qui ont une certaine instruction le goût de l'étude et des bonnes lectures ${ }^{60}$. J'entretiens l'espoir que chaque curé va donner un nouvel élan à sa bibliothèque paroissiale ${ }^{61}$."

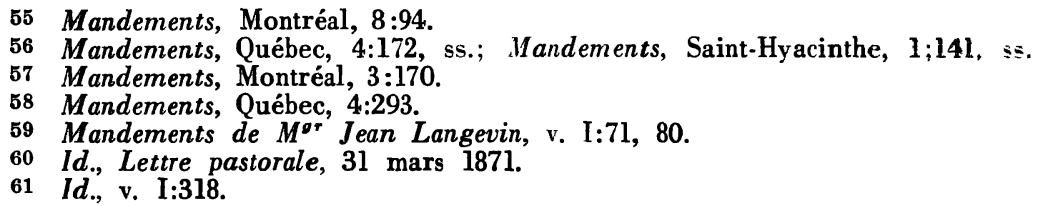


L'année suivante, d'après les rapports fournis par les curés, dixsept paroisses sur quarante-trois ont leur bibliothèque, et l'évêque exprime le désir que tous s'occupent "avec ardeur de ce sujet important $\gg 62$.

A l'occasion du troisième concile provincial, tenu en mai 1863, à l'occasion aussi du quatrième, en mai 1868, les Pères publient des lettres pastorales où ils traitent des bibliothèques paroissiales et de l'obligation où ils mettent les fidèles de seconder les efforts de leurs pasteurs dans cette matière ${ }^{63}$.

Le 24 septembre 1868 , dans une circulaire à son clergé, $\mathrm{M}^{\mathrm{gr}}$ Baillargeon annonce qu'il a pris la décision de faire imprimer un catalogue des livres qu'une bibliothèque paroissiale doit contenir. Il demande à ses curés, pour l'aider à la préparation de ce catalogue, de lui faire parvenir des suggestions : «Ayez donc la bonté de m'envoyer le plus tôt possible une liste de ceux de ces livres que vous jugerez les meilleurs et les plus utiles, surtout pour nos bons habitants des campagnes ${ }^{64}$."

Un catalogue de ce genre paraît en 1870, par les soins de la Société Saint-Vincent-de-Paul de la ville de Québec, sous le titre : Catalogue de livres recommandés par la Société Saint-Vincent-de-Paul pour les bibliothèques paroissiales. Le catalogue contient des ouvrages d'édification, de la littérature populaire sur les sciences, des traités d'agriculture et de métiers, ainsi que des romans et des recueils de contes.

De 1845 à 1870, environ, c'est donc toute une série de documents officiels où les autorités ecclésiastiques ont exposé leur intention et leur volonté de susciter et de promouvoir des bibliothèques paroissiales. Par la suite, il s'écoule une certaine période pendant laquelle le sujet apparaît plus rarement dans les mandements et les lettres des évêques; on peut noter cependant que les mises en garde à propos des mauvais livres et des lectures dangereuses continuent de manifester leurs préoccupations à ce propos.

Les bibliothèques reviennent à l'ordre du jour dans une circulaire de $M^{\mathrm{gr}}$ Bégin, coadjuteur de Québec, en date du 12 février $1894^{65}$. Il y rappelle la déclaration des Pères du deuxième Concile provincial de 1854 sur l'utilité de l'établissement de bibliothèques paroissiales. « Ces paroles ont encore toute leur utilité première ", souligne le document. Pour répondre à la demande de plusieurs curés, l'évêque annonce la publication d'une liste intitulée, Livres de choix pour les bibliothèques paroissiales, qui paraît en appendice à la circulaire : la liste comprend 525 titres d'ouvrages, dont 311 portent sur des sujets religieux. Une circulaire subséquente, le $1^{\text {er }}$ septembre 1898 , revient sur le sujet, et réfère d'ailleurs à celle de février $1894^{66}$ et donne une liste d'auteurs à l'index et dangereux.

\footnotetext{
62 Id., v. II, $\mathrm{n}^{\circ} 20$.

63 Mandements, Québec, 4:446; Mandements, Saint-Hyacinthe, 3:50, ss.

64 Mandements, Québec, 4:652.

65 Id., 8:93.

66 Mandements, Québec, 9:51; Circulaire, $1^{\text {er }}$ sept. 1898.
} 
Avec ce dernier document se termine notre quête des documents épiscopaux, publiés avant 1900 , sur la question des bibliothèques paroissiales. A parcourir leurs textes explicites, il apparaît que l'œuvre des bibliothèques paroissiales a été reconnue et prônée par la hiérarchie, papes et évêques, comme une organisation religieuse dont les évêques et les curés ont pris la direction et la responsabilité. Les documents publiés ne se sont pas limités à des considérations générales, ils comportent aussi les détails de l'organisation pratique des bibliothèques, déterminant, par exemple, comment trouver les moyens financiers néces-

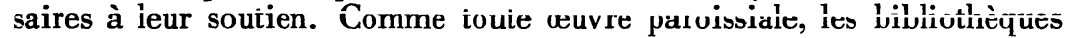
pouvaient compter sur des quêtes faites à l'église, en plus des dons faits en leur faveur et des abonnements payés par leurs abonnés.

Il n'est pas sans intérêt d'ajouter que, pour favoriser leur développement, une publication périodique a paru pendant plusieurs années, L'Echo du Cabinet de lecture paroissial. Le Cabinet de lecture paroissial, société littéraire, fondée en 1857, fut un de ces organismes par lesquels on a voulu concurrencer l'Institut Canadien de Montréal, à partir du moment où il commença ses démêlés avec $\mathbf{M}^{\mathrm{gr}}$ Bourget. En 1859, le Cabinet de lecture commença à faire paraître L'Echo du Cabinet de lecture, qui dura jusqu'en 1873. Ce fut un recueil où paraissaient les travaux littéraires présentés aux réunions du Cabinet de lecture, et destiné aux maisons d'éducation, aux instituts littéraires, aux bibliothèques paroissiales et aux familles. L'Echo du Cabinet de lecture fut, en fait, la première publication canadienne destinée, en partie du moins, aux bibliothèques paroissiales. Il n'y a pas lieu ici de rappeler la lutte qu'eut à soutenir $\mathbf{M}^{\text {gr }}$ Bourget contre les idées et les activités de l'Institut Canadien, sinon pour marquer que cette lutte s'inspira de la même conviction qui lui fit promouvoir les bibliothèques paroissiales et insister sur le danger des mauvais livres et la nécessité des bons livres.

Après avoir exposé rapidement les diverses déclarations et les mesures prises à propos des bibliothèques, il convient de se demander quels résultats pratiques elles ont $\mathrm{pu}$ amener.

Bien que notre étude porte surtout sur ce qui s'est fait au cours du $\mathrm{XIX}^{\mathrm{e}}$ siècle, il n'est pas sans utilité d'indiquer que le mouvement des bibliothèques ne s'est pas limité à cette période; en fait, il s'est prolongé bien après 1900. De nos jours encore, pour nombre de gens, dans les diverses régions de la province de Québec, la bibliothèque paroissiale a été, après l'école, la porte d'entrée dans le monde des connaissances. L'expérience qu'ils ont eue de l'utilité pratique de ce genre de bibliothèques, les bénéfices qu'ils ont pu en retirer, sont sans doute de nature impondérable, mais ils n'en sont pas moins à mettre à l'actif, ils n'entrent pas moins dans les résultats apportés par ce moyen de connaissance et de culture.

A ce témoignage, on peut ajouter celui des chiffres - quand ils sont disponibles - malgré leur sécheresse. Certains aspects des faits peuvent, grâce à eux, apparaître d'une façon objective, et quelquefois, très évocatrice de la réalité. Il faut reconnaître tout de suite, cependant, que les statistiques sur les bibliothèques paroissiales ne sont pas des 
plus abondantes ni des plus faciles à trouver. Les plus anciennes que nous ayons pu retracer remontent à 1853 et ne couvrent d'ailleurs qu'une certaine partie des années écoulées depuis.

Avant d'en faire état, et pour les apprécier justement, il y a lieu de rappeler que, dans l'intention bien explicite des évêques, les bibliothèques paroissiales ne devaient relever que de l'autorité et de la direction ecclésiastiques. Le règlement disciplinaire du deuxième Concile provincial de Québec, paru le 4 juin 1854, précise la volonté des évêques de « former des bibliothèques indépendantes de celles des commissaires d'écoles sur lesquelles les curés ne sont pas sûrs d'avoir toujours le contrôle et où, par conséquent, il pourrait se glisser des ouvrages dont la religion aurait à gémir ${ }^{67}$.

C'était précaution élémentaire, il fallait de toute évidence éviter que les bibliothèques fondées pour propager les idées saines soient diverties de leur but et ne deviennent à leur tour source de dangers. Dans bien des cas, cependant, à partir du moment où la confessionnalité des écoles fut assurée par la législation (1845), les bibliothèques paroissiales se fusionnèrent souvent avec les bibliothèques scolaires, tout en demeurant cependant sous la direction immédiate des curés ${ }^{68}$. Sans doute y eut-il certains avantages à cette façon d'agir, particulièrement en ce qui regarde les subventions qui furent accordées aux bibliothèques scolaires. A cause de cette situation, il arrive que certaines statistiques peuvent couvrir les deux genres de bibliothèques : elles n'en révèlent pas moins l'essor pris par des bibliothèques de langue française sous la direction officielle ecclésiastique.

Voici donc quelques chiffres qui peuvent donner une idée du développement des bibliothèques paroissiales :

En 1853, soit huit ans après le début officiel du mouvement, il en existait 90 , contenant 47,763 volumes.

En 1857, il en existait 96 , contenant 60,570 volumes ${ }^{69}$.

En 1860, d'après le Rapport du surintendant de l'Instruction publique, il existait environ 100 bibliothèques paroissiales, comptant 100,000 volumes ${ }^{70}$.

Certaines de ces bibliothèques étaient considérables, telle la bibliothèque de Montréal pour les pauvres classes, avec 12,000 volumes ${ }^{71}$, celle de la paroisse Notre-Dame de Québec, de 4,000 volumes, la bibliothèque paroissiale de Montréal, de 2,500. Un bon nombre atteignait le millier de volumes. On se rappelle que la liste publiée sous la direction de l'archevêque de Québec, en 1894, comportait quelque 1,225 volumes, formant ce qu'on pourrait appeler la bibliothèque-type.

Mandements, Québec, 4:167.

68 Meilleur, Mémorial, 1860 , pp. 171, 170, 172.

69 Journal of Education of Upper Canada, 13:68, 1860, Rapport du Surintendant de l'Instruction publique du Bas-Canada, 1856-1857.

70 Metlleur, Mémorial, 1860, p. 172.

71 Journal of Education of Upper Canada, 13:68, 1860. 
Des statistiques plus récentes fournissent une rétrospective de l'extension des bibliothèques paroissiales et en font connaître le prolongement depuis 1900. Elles furent publiées en 1938 par le Service de la Statistique de la province de Québec. A cette date, 333 bibliothèques avaient fait rapport. Elles contenaient 270,000 volumes et elles avaient pratiqué 440,000 prêts au cours de l'année. Par comparaison avec des statistiques relevées en 1924, le nombre des bibliothèques avait augmenté de 108.

Des bibliothèques en activité en 1938,90 avaient été fondées à une époque antérieure à 1880 et 58 , entre 1880 et 1900 .

Il semble bien qu'aux environs de 1900, et par la suite, le mouvement des bibliothèques paroissiales a connu de nouveau un essor semblable à celui des premières années, alors qu'en moins de quinze ans une centaine avaient été fondées.

Cet élan nouveau, il est contemporain de l'établissement des bibliothèques Carnegie, alors en plein essor. Les dons d'Andrew Carnegie, milliardaire philanthrope, assuraient les fonds nécessaires à la construction d'édifices pour des bibliothèques, à condition qu'elles fussent administrées par des corps publics nommés par les administrations municipales. A l'époque, l'opinion catholique, dans la province de Québec, se montra défavorable à des bibliothèques de ce genre et. de fait, aucune n'y fut construite, alors qu'elles furent acceptées dans les autres provinces ${ }^{72}$. Un bon nombre de bibliothèques paroissiales sont apparues dans les débuts du XX' siècle : 143 , de 1900 à 1925 , 116, de 1926 à 1938. Ces nouveaux établissements apportaient une preuve nouvelle, s'il en était besoin, que les autorités ecclésiastiques désiraient maintenir un système de bibliothèques populaires inspirées et dirigées par elles.

Les circonstances ont sans doute changé depuis l'époque de l'Union, alors que l'Eglise canadienne a assuré son influence dans les domaines qu'elle considérait de ses responsabilités, particulièrement celui de l'éducation, auquel se rattachent les bibliothèques. Les conditions ont aussi évolué depuis le début du siècle. Il y a lieu de reconnaître cependant que l'action de l'Eglise au sujet des bibliothèques a certainement contribué à éclairer l'opinion publique sur leur nécessité, et sur la non moindre nécessité de maintenir au premier plan, dans leur établissement et leur direction, les principes de la morale catholique.

La direction morale que l'Eglise exerçait sur les bibliothèques a été reconnue par une commission d'enquête sur les bibliothèques canadiennes. Dans le rapport que cette commission publiait en 1934, elle n'a pas manqué de noter le rôle important de l'Eglise dans la province de Québec, ainsi que la place que pouvaient occuper les bibliothèques paroissiales dans un système de bibliothèques populaires.

72 Au sujet des bibliothèques publiques et de l'opinion de la province de Quévec. cf. Th. Jolivet, Bibliothèques publiques, La Nouvelle-France, I, 1902, p. 560. ss.; Thomas Chapais, conférence prononcée le 24, octobre 1905 sur l'Apostolat des bons livres; Discours et conférences, $2^{\circ}$ série, Québec, 1913, p. 222: G.-E. Marquis, La bibliothèque publique, Québec, 1934, p. 6, note. 
Quelque vingt ans plus tard, une autre commission a donné une place aux bibliothèques paroissiales parmi les organismes qui ont pu favoriser la culture populaire au $\mathrm{XIX}^{\mathbf{e}}$ siècle.

Dans son rapport publié en 1951, la Commission royale sur lavancement des arts, des lettres et des sciences résumait ainsi l'histoire des bibliothèques paroissiales :

Si nous nous tournons vers la province de Québec, à l'époque du début du siècle, nous pouvons y observer des villes et des villages heureux; puisant dans leurs propres ressources, ils pouvaient se fournir de tout ce qui était nécessaire à la distraction et à l'instruction de leurs citoyens, sauf de livres et de journaux illustrés (p. 21).

Ce fut une période où le temps et la tranquillité ne manquaient pas pour la lecture. Dans les campagnes, la bibliothèque paroissiale, de 300 à 400 volumes, suffisait aux besoins des liseurs. On la conservait habituellement dans les armoires de la sacristie et, peu à peu, ces romans inoffensifs, ces ouvrages d'hagiographie, et quelques-uns ćtaient d'étonnants ouvrages d'érudition, s'imprégnaient d'une délicieuse odeur d'en. cens refroidi. Le curé régnait de loin sur la bibliothèque et c'est la maîtresse d'école qui en assumait le service... Sans le savoir, elle faisait déjà de l'éducation des adultes, et elle le faisait bien (p. 22).

C'est un témoignage qui résume d'une façon sommaire, et même un peu fantaisiste, une histoire longue maintenant de plus d'un siècle, mais il n'est pas sans intérêt et ne manque pas de bienveillance.

De fait, ne peut-on pas considérer que, dans la province de Québec, les bibliothèques paroissiales ont été, dans le passé, l'entreprise la plus considérable de bibliothèques destinées au peuple? Conçues d'abord comme un moyen d'apostolat religieux, elles n'en ont pas moins contribué à l'instruction et à la culture populaires. Depuis quelques années, dans la province de Québec, les administrations publiques acceptent peu à peu leur responsabilité dans ce domaine, en facilitant le développement des bibliothèques publiques. Il reste aux bibliothèques paroissiales un rôle qui leur est propre, celui de contribuer à la culture religieuse des fidèles, comme elles le font dans d'autres milieux catholiques, et c'est un domaine où elles peuvent rendre encore des services indispensables.

\section{Antonio Drolet}

Québec, P.Q.

Mai 1962 